\title{
Atividade Alelopática de Chalcona Sintética, de seus Precursores e de Cetonas e Aldeídos Relacionados
}

\author{
Allelopathic Activity of Synthetic Chalcone, Its Precursors and of Related Cetones and Aldehydes
}

\author{
BITENCOURT, H.R. ${ }^{2}$, SANTOS, L.S. ${ }^{2}$ e SOUZA FILHO, A.P.S. ${ }^{3}$
}

\begin{abstract}
RESUMO - Vários metabólitos produzidos por plantas apresentam atividade herbicida. Essa característica tem levado à possibilidade de utilizar essas substâncias como herbicidas mais integrados às atuais exigências da sociedade. Entretanto, a obtenção dessas moléculas apresenta várias limitações, sendo, dessa forma, a sintese uma alternativa. Este trabalho teve por objetivo determinar as variações na atividade alelopática da chalcona, 2,4 'dimetoxichalcona, em função dos precursores, ortoanisaldeído e 4-metoxiacetofenona, e de alterações nestes. Os bioensaios foram monitorados em condições controladas de $25{ }^{\circ} \mathrm{C}$ e fotoperíodo de 12 horas. Como plantas-teste foram utilizadas as plantas daninhas malícia (Mimosa pudica) e mata-pasto (Senna obtusifolia). A atividade alelopática foi analisada em concentrações fixas de 100, 200 e $300 \mathrm{mg} \mathrm{L}^{-1}$. A chalcona foi obtida via reação de condensação entre a 4-metoxiacetofenona e o ortoanisaldeído. Os resultados indicaram que a atividade alelopática está relacionada, em princípio, ao precursor A, 4-metoxiacetofenona. Mudanças nos precursores indicaram a possibilidade de aumentar a atividade alelopática, em especial no precursor A. Ausência do grupo $\mathrm{CH}_{3} \mathrm{O}$ propiciou redução na atividade alelopática, indicando ser importante para a atividade. A presença de dois grupamentos $\mathrm{OCH}_{3}$ no precursor da porção B da chalcona aumentou para $62 \%$ a atividade do aldeído. Esses resultados indicam que alterações de substâncias com atividades alelopáticas são uma possivivel via para enfrentar os problemas atrelados aos processos de isolamento e identificação de moléculas químicas com atividade alelopática, em espécies de plantas.
\end{abstract}

Palavras-chave: alelopatia, aleloquímico, germinação, síntese de chalcona.

ABSTRACT - Several secondary metabolites produced by plants can be used as herbicides. These products are expected to offer fewer risks to the environment and to meet social demands. However, the availability of compounds is very limited, so synthesis is an alternative. The objective of this work was to evaluate the allelopathic activity of synthetic chalcone, 2,4'-dimethoxychalcone, its precursors, 4-methoxyacetophenone (A) and ortho-anisaldehyde (B), and some of their chemical alterations. The bioassays were developed under $25{ }^{\circ} \mathrm{C}$ and photoperiod of 12 hours. The allelopathic effects of the compounds were tested on seed germination of Mimosa pudica and Senna obtusifolia weeds, under a concentration of 100, 200 and $300 \mathrm{mg} \mathrm{L}^{-1}$ of the compounds. The results indicated that precursor A (4-methoxyacetophenone) plays an important role in allelopathic activity of the chalcones. These results show that synthesis is a possible pathway, helping to overcome problems such as characterization and isolation of natural products with herbicidal activity against weed species.

Key words: allelopathy, allelochemical, germination, chalcone synthesis.

1 Recebido para publicação em 7.3.2007 e na forma revisada em 25.10.2007.

Universidade Federal do Pará, Rua Augusto Corrêa no 01, 66075-110, Belém-Pará, Brasil.

Embrapa Amazônia Oriental, Trav. Enéas Pinheiro, S/N, 66095-100, Belém-Pará, Brasil.

Planta Daninha, Viçosa-MG v. 25, n. 4, p. 747-753, 2007 


\section{INTRODUÇÃO}

Nas últimas décadas, a ciência concentrou esforços expressivos no sentido de fornecer fontes alternativas para a produção de defensivos agrícolas. Essa atitude foi motivada pelas insatisfações de ordem social em face dos problemas decorrentes do uso de produtos sintéticos, em relação à questão ambiental, e da qualidade dos alimentos utilizados na dieta dos animais, em geral, da vida silvestre e da saúde humana (Killham \& Foster, 1996; Anaya, 1999).

Uma das alternativas viáveis para fazer frente a essas questões são as moléculas químicas produzidas pelas plantas. Muitas dessas moléculas agem sobre o metabolismo das plantas, de forma semelhante aos herbicidas, constituindo-se em substitutos aos produtos sintéticos disponiveis no mercado (Kenfield et al., 1988; Strobel et al., 1991; Duke et al., 2000, 2002; Einhellig, 2001). Paralelamente, as informações disponíveis apontam para existência de similaridades entre os sitios moleculares de ação dos produtos sintéticos com as moléculas naturais (Duke \& Abbas, 1995). Adicionalmente, as moléculas naturais possuem especificidades que representam pouco risco para os recursos naturais e para os interesses da sociedade; nos trabalhos de Cutler (1988), Duke \& Lydon (1987) e Henkel et al. (1999) esses aspectos são amplamente discutidos.

Entretanto, no tocante aos procedimentos metodológicos utilizados no isolamento, na identificação e na caracterização da atividade alelopática de substâncias químicas produzidas pelas plantas, além de demorados e onerosos, em muitos casos os resultados obtidos fornecem aleloquímicos com baixa atividade inibitória da germinação de sementes e do desenvolvimento da radícula de espécies de plantas receptoras, ou, então, as quantidades obtidas são insuficientes para realização dos bioensaios, permitindo, apenas, a realização dos procedimentos de identificação. Bons exemplos dessas implicações são encontrados nos trabalhos de Fonseca (2005), Lobo (2004) e Pereira (2003). Outro fator que limita os avanços é o número de centros quirais na molécula, o que dificulta ou, em determinados casos, inviabiliza sua sintese. Uma alternativa para esse problema seria a manipulação da molécula química de determinadas substâncias com atividades alelopáticas já comprovadas mediante bioensaios, sem que os procedimentos empregados redundem no comprometimento das propriedades biológicas, que as tornam importantes fontes alternativas para enfrentar os problemas que surgem em decorrência da aplicação dos herbicidas disponiveis no mercado.

Os flavonóides, pela sua importância entre as classes de substâncias químicas com atividade alelopática (Moreland \& Novitzky, 1987; Rice, 1987), podem servir de base para derivações de sua molécula. As chalconas, por não possuírem nenhum centro quiral e serem as suas precursoras biossintéticas, podem servir de base para estudos de derivações de sua molécula.

No presente trabalho foram testadas duas hipóteses: a atividade alelopática de uma dada molécula química depende mais de um dos seus precursores (componentes) do que da molécula completa; e é possivel aumentar a atividade alelopática de uma dada molécula via alterações dos seus precursores. Dessa forma, este trabalho teve por objetivo determinar as variações na atividade alelopática da chalcona SC, 2E- 3-(2-metoxifenil)-1-(4-metoxifenil)prop-2-en-1-ona (2,4'-dimetoxi-chalcona), dos seus precursores, 4-metoxiacetofenoa e ortoanisaldeído, e das cetonas (A1 e A2) e aldeídos (B1, B2 e B3) relacionados.

\section{MATERIAL E MÉTODOS}

\section{Procedimento de sintese e caracterização da substância química}

A substância SC foi obtida por reação de condensação em meio básico (Figura 1), utilizando-se a metodologia que se segue (Hathaway, 1987). Em um balão de fundo chato e boca esmerilhada $(125 \mathrm{~mL})$ mantido em banho de gelo, foram adicionados $1,2 \mathrm{~g}(8 \mathrm{mmol})$ de 4-metoxiacetofenona (PA), $2 \mathrm{~mL} \mathrm{(16} \mathrm{mmol)}$ de ortoanisaldeído (PB), $15 \mathrm{~mL}$ de etanol e 10 mL de solução de hidróxido de sódio 10\%. A mistura reacional foi mantida em agitação magnética a $40^{\circ} \mathrm{C}$ por $30 \mathrm{~min}$. Posteriormente, foi deixada em freezer durante 48h. Após esse período, procedeu-se à decantação e a fase 
inferior oleosa foi lavada com água destilada a frio. O material oleoso foi identificado como $\mathrm{SC}$ (1,7 g; $78 \%$ de rendimento).

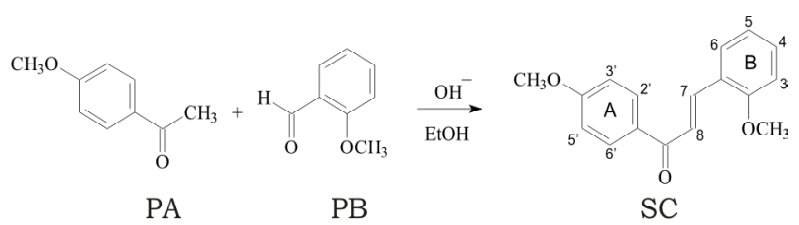

Figura 1 - equação química da substância sintetizada

A confirmação da estrutura química de SC foi feita por ressonância magnética nuclear de hidrogênio. No espectro de $\mathrm{RMN}^{1} \mathrm{H}$ de SC (300 MHz; $\mathrm{CDCl}_{3}$ ), podem ser observados os sinais relativos aos dupletos dos hidrogênios 8 $(\delta 7,62)$ e $7(\delta 8,10)$ como sendo um sistema AB, $J_{\text {trans }}=16 \mathrm{~Hz}$. Para o anel A observa-se o sistema AA'BB', relativo aos sinais dos hidrogênios 2', 6' $(\delta 8,05-8,02)$ e 3', 5' $(\delta 6,95-6,98)$. Quanto ao anel B, observam-se os sinais relativos a H-6 (d; $\mathrm{J}_{\text {orto }}=8,4 \mathrm{~Hz} ; \delta 6,93$ ), $\mathrm{H}-5$ (ddd; $\mathrm{J}_{\text {meta }}=1,8 \mathrm{~Hz}$; $\left.\mathrm{J}_{\text {orto }}=7,5 \mathrm{~Hz} ; \mathrm{J}_{\text {orto }}=8,4 \mathrm{~Hz} ; \delta 7,36\right), \mathrm{H}-4$ (dd; $\mathrm{J}_{\text {orto }}=$ $\left.7,5 \mathrm{~Hz} ; \mathrm{J}_{\text {orto }}=7,8 \mathrm{~Hz} ; \delta 6,98\right)$ e $\mathrm{H}-3\left(\mathrm{dd} ; \mathrm{J}_{\text {meta }}=\right.$ $1,8 \mathrm{~Hz} ; \mathrm{J}_{\text {orto }}=7,8 \mathrm{~Hz} ; \delta 7,63$ ), além de dois simpletos relativos aos sinais dos hidrogênios das duas metoxilas em $\delta 3,90$ e $\delta 3,87$.

\section{Avaliação da atividade alelopática das substâncias químicas}

Os bioensaios de germinação foram desenvolvidos em câmaras de germinação, com temperatura constante de $25{ }^{\circ} \mathrm{C}$ e fotoperiodo de 12 horas, com três repetições. A germinação foi monitorada em periodos de cinco dias, com contagens diárias e eliminação das sementes germinadas. Foram consideradas sementes germinadas aquelas que apresentavam extensão radicular igual ou superior a $2,00 \mathrm{~mm}$ (Juntila, 1976; Duram \& Tortosa, 1985). Cada placa de Petri, de $9,0 \mathrm{~cm}$ de diâmetro, forrada com um disco de papel-filtro qualitativo, recebeu 10 sementes.

\section{Outros procedimentos experimentais}

As avaliações das substâncias foram feitas nas concentrações de 100, 200 e $300 \mathrm{mgL}^{-1}$, tendo como solvente o clorofórmio. Cada placa de Petri de 9,0 cm de diâmetro recebeu $3 \mathrm{~mL}$ de solução. O material avaliado foi adicionado apenas uma vez, no início de cada bioensaio, sendo adicionada, a partir de então, apenas água destilada, sempre que necessário. Após adição das soluções, deixou-se evaporar o solvente e adicionaram-se 3,0 $\mathrm{mL}$ de água destilada, mantendo-se, dessa forma, a concentração original.

Como plantas receptoras, foram utilizadas as espécies de plantas daninhas de área de pastagem cultivada malícia (Mimosa pudica) e mata-pasto (Senna obtusifolia). As sementes foram coletadas em áreas de pastagens cultivadas do municipio de Castanhal-PA e passaram por um processo de limpeza e superação da dormência, via imersão em ácido sulfúrico concentrado (Souza Filho et al., 1998).

\section{Análise estatística}

O delineamento experimental foi o inteiramente casualizado, com três repetições, tendo como tratamento testemunha água destilada. Os dados foram submetidos à análise de variância pelo teste $\mathrm{F}$ e as médias comparadas pelo teste de Tukey (5\%). Os dados foram analisados utilizando-se o programa estatístico SAS (SAS, 1989).

\section{RESULTADOS E DISCUSSÃO}

\section{Avaliação da atividade alelopática das substâncias químicas}

Na Tabela 1 são apresentados os efeitos alelopáticos da chalcona SC $(2,4$ '-dimetoxichalcona) e de seus precursores. Os resultados indicam que a substância, em si, possui atividade alelopática em nivel de inibição da germinação das sementes de malícia e matapasto, em 58 e $48 \%$, res pectivamente, na concentração de $300 \mathrm{mgL}^{-1}$. Quando se analisam comparativamente os efeitos alelopáticos inibitórios promovidos pela molécula da substância e pelos precursores, observa-se que A promoveu inibições superiores às efetivadas pela molécula completa e pelo precursor B, praticamente em todas as concentrações testadas. Quando se considera o valor de 50\% de inibição como indicador satisfatório para avaliar as potencialidades alelopáticas de um dado aleloquímico (Dudai et al., 1999), 
Tabela 1 - Análise da atividade alelopática da substância química chalcona, sintetizada, e de seus precursores A e B. Dados expressos em percentual de inibição em relação ao tratamento testemunha - água destilada

\begin{tabular}{|c|c|c|c|c|c|c|c|}
\hline \multirow{2}{*}{ Estrutura química } & \multirow{2}{*}{ Código } & \multicolumn{3}{|c|}{ Malícia } & \multicolumn{3}{|c|}{ Mata-pasto } \\
\hline & & $100 \mathrm{mg} \mathrm{L}^{-1}$ & $200 \mathrm{mg} \mathrm{L}^{-1}$ & $300 \mathrm{mg} \mathrm{L}^{-1}$ & $100 \mathrm{mg} \mathrm{L}^{-1}$ & $200 \mathrm{mg} \mathrm{L}^{-1}$ & $300 \mathrm{mg} \mathrm{L}^{-1}$ \\
\hline & $\mathrm{SC}$ & $30 \mathrm{Bc}$ & $40 \mathrm{Bb}$ & $58 \mathrm{Ba}$ & $10 \mathrm{Ac}$ & $26 \mathrm{Ab}$ & $48 \mathrm{Ba}$ \\
\hline $\mathrm{CH}_{3} \mathrm{O}$ & PA & $38 \mathrm{Ac}$ & $54 \mathrm{Ab}$ & $68 \mathrm{Aa}$ & $8 \mathrm{Ac}$ & $26 \mathrm{Ab}$ & $53 \mathrm{Aa}$ \\
\hline & PB & $10 \mathrm{Cc}$ & $15 \mathrm{Cb}$ & $21 \mathrm{Ca}$ & $3 \mathrm{Bc}$ & $10 \mathrm{Bb}$ & $21 \mathrm{Ca}$ \\
\hline $\mathrm{OCH}_{3}$ & & & & & & & \\
\hline
\end{tabular}

Médias seguidas de letras iguais, maiúsculas nas colunas e minúsculas nas linhas, dentro de cada planta receptora, não diferem pelo teste de Tukey $(5 \%)$.

$\mathrm{SC}=$ substância completa, $\mathrm{PA}=$ precursor $\mathrm{A}$ da molécula e $\mathrm{PB}=$ precursor $\mathrm{B}$ da molécula.

observase que inibições dessa magnitude foram alcançadas apenas na concentração de $300 \mathrm{mg} \mathrm{L}^{-1}$ e apenas para a planta receptora malícia. Já para o precursor A, esse valor foi atingido nas concentrações de 200 e $300 \mathrm{mg} \mathrm{L}^{-1}$, para malícia, e $300 \mathrm{mg} \mathrm{L}^{-1}$, para mata-pasto. As inibições efetivadas pelo precursor B foram bem inferiores às da molécula completa e do precursor A. Esses resultados indicam que a atividade alelopática da substância em teste está, basicamente, associada aos efeitos do fragmento A. Esses dados confirmam a primeira hipótese levantada no presente trabalho.

Em razão dos resultados obtidos e visando atingir a segunda hipótese aventada, analisou-se a atividade alelopática dos dois fragmentos (A e B), em relação às modificações de suas estruturas químicas. Nesse sentido, os dados da Tabela 2 mostram os efeitos alelopáticos inibitórios do fragmento A e das alterações de sua molécula sobre a germinação de sementes das duas plantas daninhas. A proposta de eliminação do grupo $\mathrm{CH}_{3} \mathrm{O}$, do carbono $\mathrm{C} 4$ (A1), promoveu redução da atividade alelopática inibitória da molécula. Já a substituição do grupo $\mathrm{CH}_{3} \mathrm{O}$ por $\mathrm{OH}$ não promoveu qualquer alteração na atividade da molécula quando se analisam os efeitos sobre a germinação das sementes de malícia, porém ganhos, embora em baixa magnitude, foram auferidos em relação à atividade inibitória sobre a germinação das sementes de mata-pasto. Aparentemente, a presença de um grupamento no carbono $\mathrm{C} 4$ é importante para a atividade alelopática da molécula, mas não há diferença em relação ao $\mathrm{CH}_{3} \mathrm{O}$ e ao $\mathrm{OH}$. Paralelamente, o caráter de aumento da atividade inibitória em relação ao aumento da concentração não se modifica no que se refere às alterações efetuadas.

Com relação ao precursor B, que apresentou menor atividade alelopática inibitória com relação à molécula inteira e ao fragmento $\mathrm{A}$, os dados indicaram que, das três alterações propostas (Tabela 3), apenas a A3 propiciou aumento da atividade alelopática $(\mathrm{p}<0,05)$ expressivo - da ordem de $62 \%$ na atividade em relação à molécula inteira -, embora esse efeito tenha a limitação de não ter a mesma expressão em relação aos efeitos sobre a germinação das sementes de mata-pasto. A eliminação do grupo $\mathrm{OCH}_{3}$, do carbono $\mathrm{C} 2$, redundou 
Tabela 2 - Análise da atividade alelopática do precursor A da substância química chalcona sobre a germinação de sementes de duas plantas daninhas. Dados expressos em percentual de inibição em relação ao tratamento testemunha - água destilada

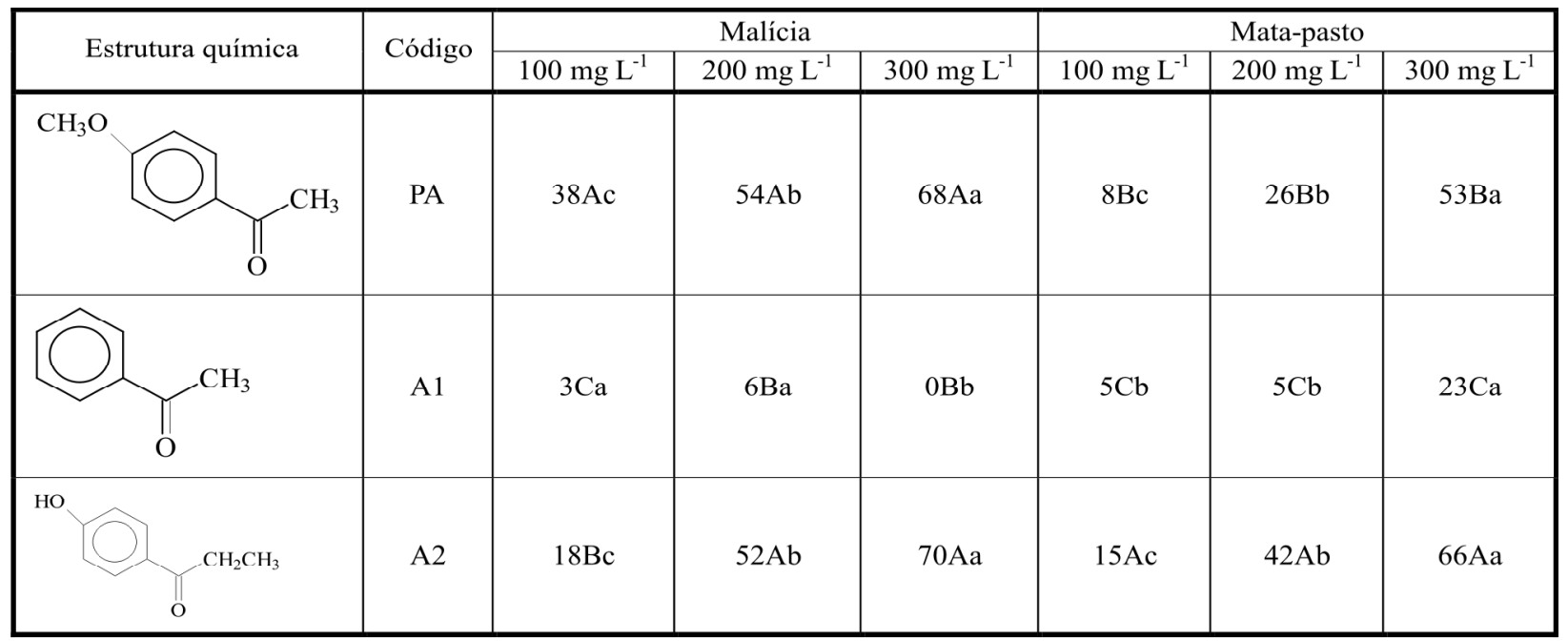

Médias seguidas de letras iguais, maiúsculas nas colunas e minúsculas nas linhas, dentro de cada planta receptora, não diferem pelo teste de Tukey (5\%).

$\mathrm{PA}=$ precursor A da molécula original, $\mathrm{A} 1$ = alteração 1 da molécula do precursor A e A2 = alteração 2 da molécula do precursor A.

Tabela 3 - Análise da atividade alelopática do precursor B da substância química chalcona sobre a germinação de sementes de duas plantas daninhas. Dados expressos em percentual de inibição em relação ao tratamento testemunha - água destilada

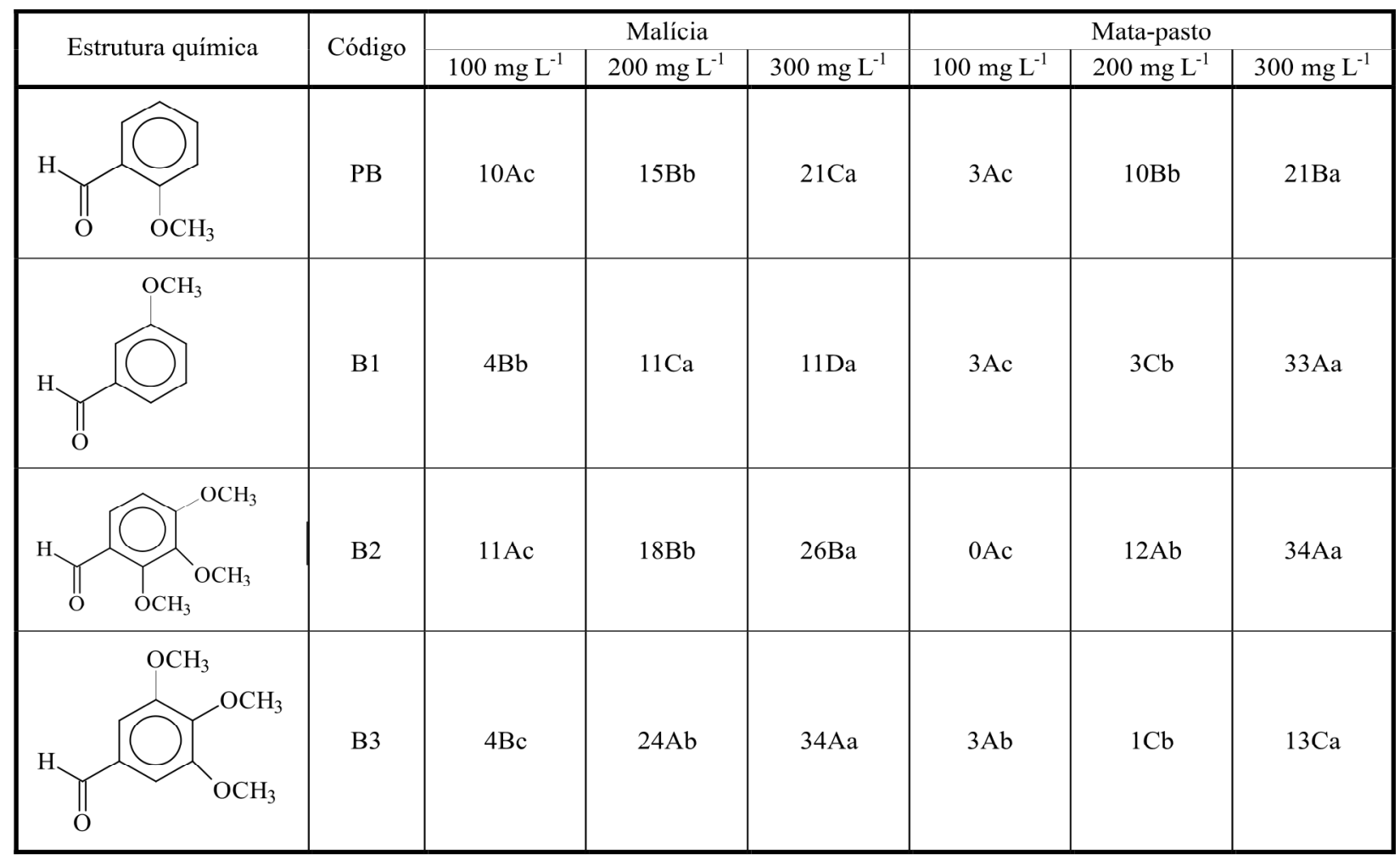

Médias seguidas de letras iguais, maiúsculas nas colunas e minúsculas nas linhas, dentro de cada planta receptora, não diferem pelo teste de Tukey $(5 \%)$.

$\mathrm{PB}=$ precursor $\mathrm{B}$ da molécula original, $\mathrm{B} 1$ = alteração 1 da molécula do precursor $\mathrm{B}, \mathrm{B} 2$ = alteração 2 da molécula do precursor $\mathrm{B}$ e B3 = alteração 3 da molécula do precursor B. 
em redução da atividade alelopática inibitória, indicando que a presença de um grupamento, nesse carbono, é determinante para a atividade da molécula; tanto assim que, em todas as derivações onde havia um grupamento nesse carbono, a atividade alelopática foi superior. À semelhança dos demais efeitos observados, o fator concentração praticamente não foi alterado pelas alterações propostas na molécula da chalcona. Os resultados obtidos no presente trabalho fornecem subsídios que dão suporte para confirmar a segunda hipótese aventada no presente trabalho.

As informações disponíveis na literatura, analisando as variações na atividade alelopática de uma dada molécula, são extremamente limitadas. Em um dos poucos trabalhos nesse sentido, Souza Filho et al. (2006), avaliando as variações nos efeitos da substância química titonina sobre a germinação e o desenvolvimento das plantas daninhas malícia e matapasto, verificaram que a acetilação da molécula promoveu aumentos significativos na sua atividade alelopática inibitória.

De qualquer forma, esse resultado sinaliza que a alteração das moléculas químicas com atividades alelopáticas é uma alternativa viável para se aumentar a atividade alelopática da molécula e fazer frente aos problemas que limitam as pesquisas de isolamento e identificação de moléculas químicas com atividade alelopática.

\section{LITERATURA CITADA}

ANAYA, A. L. Allelopathy as a tool in the management of biotic resources in agroecosystems. Crit. Rev. Plant Sci., v. 18, n. 6, p. 697-739, 1999.

CUTLER, H.G. Perspectives on discovery of microbial phytotoxins with herbicidal activity. Weed Technol., v. 2, p. 525-532, 1988.

DUDAI, N. et al. Essential oils as allelochemicals and their potential use as bioerbicides. J. Chem. Ecol., v. 25, n. 5, p. 1079-1089, 1999.

DUKE, O. S. et al. Chemical from nature for weed managemnt. Weed Sci., v. 50, p. 138-151, 2002.

DUKE, O. S.; ABBAS, H. K. Natural products with potential use as herbicides. In: INDERJIT; DAKSHINI, K. M. N.; EINHELLIG, F. A. Allelopathy: organisms, processes and applications. Washington: American Chemical Society, 1995. p. 348-362. (ACS. Symposium Series, 582).
DUKE, O. S.; LYNDON, J. Herbicides from natural compounds. Weed Technol., v. 1, p. 122-128, 1987.

DUKE, O. S.; ROMAHNI, J. G.; DAYAN, F. E. Natural products as sources for a new mechanism of herbicidal action. Crop Prod., v. 19, p. 583-589, 2000.

DURAM, J. M.; TORTOSA, M. E. The effect of mechanical and chemical scarification on germination of charlock (Sinapsis arvensis L.) seeds. Seed Sci. Technol., v. 13, n. 1, p. 155-163, 1985.

EINHELLIG, F. A. The physiology of allelochemical action: clues and views. In: REIGOSA, M. J.; BONJOCH, N. T. (Eds.). EUROPEAN OBCD ALLELOPATHY SYMPOSIUM: PHYSIOLOGICAL ASPECTS OS ALLELOPATHY, 1., Vigor Spain, 2001. Proceedings... 2001. p. 3-25.

FONSECA, M. L. Potencial alelopático de plantas da família Leguminosae: investigação de aleloquímicos de Parkia perndula . 2005. 121 f. Dissertação (Mestrado em Química) - Universidade Federal do Pará, Belém, 2005.

HATHAWAY, B. A. An aldol condensation experiment using a number of aldehydes and ketones. J. Chem. Educ., v. 64, n. 4, p. 367-368, 1987.

HENKEL, T. et al. Statistical investigation into the structural complementary of natural products and synthetic compounds. Ang. Chem. Intern. Ed. English, v. 38, p. 643-647, 1999.

JUNTILA, O. Seed and embryo germination in S. vulgaris and $S$. reflexa as affected by temperature during seed development. Physiol. Plant., v. 29, p. 264-268, 1976.

KENFIELD, D. et al. Potential new herbicides: phytotoxins from plant pathogens. Weed Technol, v. 2, p. 519-524, 1988.

KILLHAM, K.; FOSTER, R. Soil ecology. Cambridge: University Press, 1996. 242 p.

LOBO, L. T. Estudo das propriedades alelopáticas de plantas: investigação de aleloquímicos de Tachigali mymercvophyla (Leguminosae). 2004. 134 f. Dissertação (Mestrado em Química) - Universidade Federal do Pará, Belém, 2004.

MORELAND, D. E.; NOVITZKY, W.P. Effects of phenolic acids, coumarins, and flavonoids on isolates chloroplasts and mitochondria. In: WALLER, G. R. (Ed.). Allelochemicals : role in agriculture and forestry. Washington: American Chemical Society, 1987. p. 247-261.

PEREIRA, A. A. G. Brachiaria humidicola (Rendel) Schweickerdt (Poaceae) - composição fitoquímica dos extratos polares e análise da atividade alelopática . 2003. 109 f. Dissertação (Mestrado em Química) - Universidade Federal do Pará, Belém, 2003. 
RICE, E. L. Allelopathy: an overview. In: WALLER, G. R. (Ed.). Allelochemical: role in agriculture and forestry. Washington: American Chemical Society, 1987. p. 7-22.

SOUZA FILHO, A. P. S.; DUTRA, S.; SILVA, M. A. M. M Métodos de superação da dormência de sementes de plantas daninhas de pastagens cultivadas da Amazônia. Planta Daninha, v. 16, n. 1, p. 3-11, 1998.
SOUZA FILHO, A. P. S.; BORGES, F. C.; SANTOS, L. S. Análise comparativa dos efeitos alelopáticos das substâncias químicas titonina e titonina acetilada. Planta Daninha, v. 24. n. 2, p. 209-210, 2006.

STATISTICAL ANALYSIS SYSTEM- SAS. User's guide. Version 6.4. Cary: 1998. 846 p.

STROBEL, G. et al. Phytotoxins as potential herbicides. Experientia, v. 47, n. 8, p. 819-826, 1991. 